\title{
Amplification of in-plane seismic ground motion by group cavities in layered half-space (II): with saturated poroelastic soil layers*
}

\author{
Jianwen Liang ${ }^{1,2, *}$ Ji Zhang ${ }^{1,2}$ and Zhenning Ba ${ }^{1,2}$ \\ ${ }^{1}$ Department of Civil Engineering, Tianjin University, Tianjin 300072, China \\ 2 Tianjin Key Laboratory of Civil Engineering Structures and New Materials, Tianjin 300072, China
}

\begin{abstract}
As the continuation study on amplification of in-plane seismic ground motion by underground group cavities in layered half-space, this study extends to the case of poroelastic half-space with dry poroelastic and saturated poroelastic soil layers. The influence of poroelastic layers on the amplification of seismic ground motion is studied both in frequency domain and time domain using indirect boundary element method (IBEM). It is shown that for the example of a saturated poroelastic site in Tianjin under the excitation of Taft wave and El Centro wave, the amplification of seismic ground motion in poroelastic case is slightly smaller than that in the elastic case, and the amplification of PGA (peak ground acceleration) and its PRS (peak response spectrum) can be increased up to $38.8 \%$ and $64.6 \%$; the predominant period of response spectra in poroelastic case becomes shorter to some extent compared with that in the elastic case. It is suggested that the effect of underground group cavities in poroelastic half-space on design seismic ground motion should be considered.
\end{abstract}

Key words: underground group cavity; dry poroelastic; saturated poroelastic; amplification; inplane; seismic ground motion; indirect boundary element method (IBEM)

CLC number: P315.9 Document code: A

\section{Introduction}

Amplification of out-of-plane or in-plane seismic ground motion by group cavities in layered half-space both in frequency domain and time domain were presented by Liang et al. (2012a, b), and it was shown that, group cavities may have significant amplification to seismic ground motion, and group cavities may also affect the spectrum of seismic ground motion.

It should be pointed that, engineering sites are often saturated in coastal area due to high ground water level, e.g., Tianjin, China, and the most common case is that the soil layer over water level is dry poroelastic, and the below is saturated poroelastic, while the bedrock is elastic.

As a continuation study of Liang et al. (2012b),

\footnotetext{
* Received 2 June 2012; accepted in revised form 16 July 2012; published 10 August 2012.

* Corresponding author. e-mail: liang@tju.edu.cn

(c) The Seismological Society of China, Institute of Geophysics,

China Earthquake Administration, and Springer-Verlag Berlin Heidelberg 2012
}

this paper studies both frequency-domain and timedomain amplification of seismic ground motion by group cavities in layered half-space with dry poroelastic and saturated poroelastic soil layers, using indirect boundary element method (IBEM) combined with the exact dynamic-stiffness matrix (Liang and You, 2004) of a poroelastic half-space and the dynamic Green's function (Liang and You, 2005) for the distributed loads or pore pressure acting on an inclined line in a poroelastic layered half-space. The purpose of this study is to explore the influence of pore liquid on the amplification of seismic ground motion by group cavities, and to provide theoretical foundation for microzonation of design seismic ground motion in coastal area.

\section{Method}

Figure 1 shows the 2-D plane strain model, in which, two circular cavities of radius $a$ and of depth $d$ with interval $B$ are located in layered half-space. The thickness of soil layers over bedrock (half-space) is $H$; the soil layers over water level is dry poroelastic, and the below saturated poroelastic, while the bedrock is elas- 
tic. The layered half-space is excited by seismic waves incident from the bedrock with angle $\theta$.

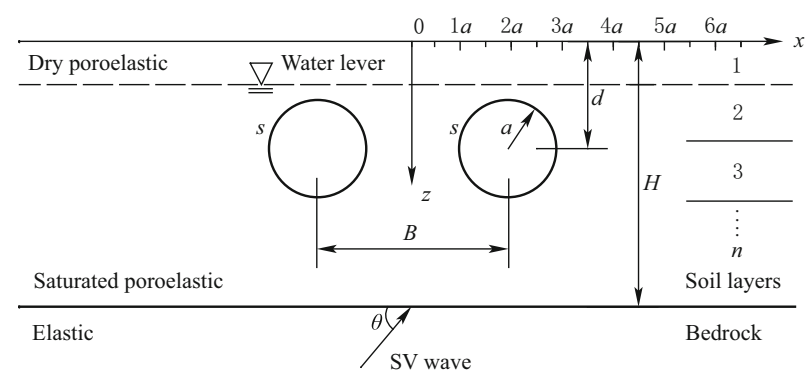

Figure 1 The model of group cavities in layered poroelastic half-space for excitation of in-plane SV waves.

The indirect boundary element method (IBEM) is used in this study. The displacements of the solid frame, the displacement of the pore fluid with respect to the solid frame, the stresses in the solid frame, and the pore pressure at the surface of the cavities are determined by the calculation of the free-field response. Then, the fictitious distributed loads and pore pressure are applied at the surface of the cavities in the free field, and the corresponding dynamic Green's functions for the displacements and stresses are obtained. Next, the amplitudes of the fictitious loads are determined by the boundary conditions, and the dynamics response can be calculated by the superposition of the response of the free field and the response due to the fictitious loads.

The exact dynamic-stiffness matrix (Liang and You, 2004) of a poroelastic half-space and the dynamic Green's function (Liang and You, 2005) for the distributed loads or pore pressure acting on an inclined line in a poroelastic layered half-space are used in this study. The details will be briefly summarized here. On the basis of Biot's equation, the general solution of the wave equation can be deduced by introducing the wave potential functions, allowing the exact dynamic-stiffness matrices of both a poroelastic soil layer and a poroelastic half-space to be derived. The global stiffness matrix of a multi-layered site is then assembled by the layered and half-space stiffness matrices while considering the boundary conditions at the interfaces between adjacent soil layers and between the bottom layer and the half-space. Using the integral transform method, the dynamic Green's functions of a distributed load or pore pressure acting on the horizontal, vertical and inclined lines in a poroelastic layered half-space can then be derived. This formalism allows the key problems in wave propagation and dynamic soil-structure interaction in poroelasticity to be resolved using the indirect boundary element method. This approach has the following advantages: all parameters in the dynamic-stiffness matrices have explicit physical meanings, and depending on the given parameters, the stiffness matrices can be reduced to either the dry poroelastic or the elastic case.

\subsection{Biot's theory}

The displacement of the solid frame and the fluid displacement relative to the solid frame are denoted by $u_{i}$ and $w_{i}(i=x, y)$, respectively, then the constitutive relationship (Biot, 1941) of a homogeneous poroelastic medium can be expressed as

$$
\begin{gathered}
\sigma_{i j}=2 \mu \varepsilon_{i j}+\lambda \delta_{i j} e-\alpha \delta_{i j} p \quad i, j=x, y, \\
p=-\alpha M u_{i, i}-M w_{i, i}
\end{gathered}
$$

where $\sigma_{i j}$ is the total stress component of the bulk material; $\varepsilon_{i j}$ and $e$ are strain components and dilatation of the solid frame, respectively; $\lambda$ and $\mu$ are Lame constants of the bulk material; $p$ is pore pressure; $\delta_{i j}$ is Kronecker delta; and $\alpha$ and $M$ are Biot's parameters describing compressibility of the two-phased material and $0 \leq \alpha \leq 1$ and $0 \leq M<\infty$.

The equations of motion (Biot, 1962) of a homogeneous poroelastic medium can be expressed in terms of displacements $u_{i}$ and $w_{i}$ as

$$
\mu u_{i, j j}+\left(\lambda+\alpha^{2} M+\mu\right) u_{j, j i}+\alpha M w_{j, j i}=\rho \ddot{u}_{i}+\rho_{\mathrm{f}} \ddot{w}_{i}
$$

and

$$
\alpha M u_{j, j i}+M w_{j, j i}=\rho_{\mathrm{f}} \ddot{u}_{i}+m \ddot{w}_{i}+b \dot{w}_{i},
$$

where $\rho=(1-n) \rho_{\mathrm{s}}+n \rho_{\mathrm{f}}, \rho_{\mathrm{s}}$ and $\rho_{\mathrm{f}}$ are mass density of the solid grain and the pore fluid, respectively; $n$ is porosity of the solid frame; $b$ is a parameter that accounts for the internal friction due to the relative motion between the solid frame and the pore fluid, and $b=0$ if the internal friction is neglected; and $m$ is a density-like parameter depending on $\rho_{\mathrm{f}}$ and geometry of the pores.

\subsection{Free-field response of a poroelastic layered \\ half-space}

By assembling the dynamic-stiffness matrices (Liang and You, 2004) of dry poroelastic and saturated poroelastic soil layers and elastic bedrock half-space, the motion equation of a layered poroelastic half-space can be expressed as

$$
\boldsymbol{S}_{\mathrm{P} 1-\mathrm{P} 2-\mathrm{SV}} \boldsymbol{U}=\boldsymbol{Q},
$$

where $\boldsymbol{S}_{\mathrm{P} 1-\mathrm{P} 2-\mathrm{SV}}$ is the global stiffness matrix of the layered half-space, $\boldsymbol{U}$ is the vector of the displacement, and $Q$ is the vector of the external loads. Solving this equation gives the free-field responses, which consists of 
the in-plane free-field displacements of the solid frame in the $x$ - and $z$-directions, the displacement of the pore fluid with respect to the solid frame, the stresses of the solid frame in the $x$ - and $z$-directions, and the pore pressure on the surface of the cavities.

\subsection{Dynamic Green's functions}

Dynamic Green's function denotes the dynamic response at any point in the free field (layered half-space) when a unit load is applied somewhere in the free field. We use dynamic Green's function (Liang and You, 2005) for the distributed loads or pore pressure acting on an inclined line in a poroelastic layered half-space in this study, therefore, no singularity will be resulted.

At first, the two interfaces in each layer are assumed to be fixed, and the corresponding reaction forces are calculated. The directions of these forces are then reversed, and they are applied as loads to determine the response. The total response can then be obtained by adding the result of the analysis of fixed layers. More details can be found in Liang and You (2005).

The displacements and stresses of the scattered field can be expressed as

$$
\left\{\begin{array}{l}
U_{x \mathrm{~g}} \\
U_{z \mathrm{~g}}
\end{array}\right\}=\boldsymbol{g}_{u \mathrm{~d}}(s)\left\{\begin{array}{l}
p(s) \\
r(s) \\
q(s)
\end{array}\right\}
$$

and

$$
\left\{\begin{array}{c}
t_{x \mathrm{~g}}(s) \\
t_{z \mathrm{~g}}(s) \\
t_{p \mathrm{~g}}(s)
\end{array}\right\}=\boldsymbol{g}_{t \mathrm{~d}}(s)\left\{\begin{array}{l}
p(s) \\
r(s) \\
q(s)
\end{array}\right\}
$$

where $\boldsymbol{g}_{u \mathrm{~d}}(s)$ and $\boldsymbol{g}_{t \mathrm{~d}}(s)$ are the dynamic Green's functions for the drained boundary. In equations (6) and (7), the subscript ' $\mathrm{g}$ ' in the displacement terms $\left\{U_{x \mathrm{~g}}, U_{z \mathrm{~g}}\right\}^{\mathrm{T}}$ and the stress terms $\left\{t_{x \mathrm{~g}}(s), t_{z \mathrm{~g}}(s) t_{\mathrm{pg}}(s)\right\}^{\mathrm{T}}$ indicates results due to the fictitious loads. The elements in the vector $\{p(s), r(s), q(s)\}^{\mathrm{T}}$ are the fictitious loads in the $x$ and $z$-directions and the fictitious pore pressure along the surface of cavities.

Similarly,

$$
\left\{\begin{array}{l}
U_{x \mathrm{~g}} \\
U_{z \mathrm{~g}}
\end{array}\right\}=\boldsymbol{g}_{u \mathrm{u}}(s)\left\{\begin{array}{l}
p(s) \\
r(s) \\
q(s)
\end{array}\right\}
$$

and

$$
\left\{\begin{array}{l}
t_{x \mathrm{~g}}(s) \\
t_{z \mathrm{~g}}(s) \\
w_{\mathrm{g}}(s)
\end{array}\right\}=\boldsymbol{g}_{t \mathrm{u}}(s)\left\{\begin{array}{l}
p(s) \\
r(s) \\
q(s)
\end{array}\right\}
$$

with $\boldsymbol{g}_{u \mathrm{u}}(s)$ and $\boldsymbol{g}_{t \mathrm{u}}(s)$ being the dynamic Green's functions for the undrained boundary.

\subsection{Boundary conditions}

The conditions for the drained boundary of the cavities can now be expressed as

$$
\int_{\mathrm{s}} \boldsymbol{W}^{\mathrm{T}}(s)\left(\left\{\begin{array}{c}
t_{x \mathrm{~g}}(s) \\
t_{z \mathrm{~g}}(s) \\
t_{\mathrm{pg}}(s)
\end{array}\right\}+\left\{\begin{array}{c}
t_{x \mathrm{f}}(s) \\
t_{z \mathrm{f}}(s) \\
t_{\mathrm{pf}}(s)
\end{array}\right\}\right) \mathrm{d} s=0
$$

with the weighting function $\boldsymbol{W}(s)$. If $\boldsymbol{W}(s)$ takes the value 1 in a single element and zero in all the others, the integral can be evaluated over each element respectively. Substituting equation (7) into equation (10) gives

$$
\boldsymbol{T}_{\mathrm{p}}\left\{\begin{array}{l}
p(s) \\
r(s) \\
q(s)
\end{array}\right\}=\boldsymbol{T}_{\mathrm{f}}
$$

with

$$
\boldsymbol{T}_{\mathrm{p}}=\int_{\mathrm{s}} \boldsymbol{W}^{\mathrm{T}}(s) \boldsymbol{g}_{t \mathrm{~d}}(s) \mathrm{d} s
$$

and

$$
\boldsymbol{T}_{\mathrm{f}}=-\int_{\mathrm{s}} \boldsymbol{W}^{\mathrm{T}}(s)\left\{t_{x \mathrm{f}}(s), t_{z \mathrm{f}}(s), t_{\mathrm{pf}}(s)\right\}^{\mathrm{T}} \mathrm{d} s .
$$

Combining the solutions of equations (11) and (6), the surface displacements become

$$
\left\{\begin{array}{l}
U_{x} \\
U_{z}
\end{array}\right\}=\left\{\begin{array}{l}
U_{x \mathrm{f}} \\
U_{z \mathrm{f}}
\end{array}\right\}+\boldsymbol{g}_{u \mathrm{~d}}(s) \boldsymbol{T}_{\mathrm{p}}^{-1} \boldsymbol{T}_{\mathrm{f}}
$$

In a similar way, the conditions for the undrained boundary of the cavities can be expressed as

$$
\int_{s} \boldsymbol{W}^{\mathrm{T}}(s)\left(\left\{\begin{array}{c}
t_{x \mathrm{~g}}(s) \\
t_{z \mathrm{~g}}(s) \\
w_{\mathrm{g}}(s)
\end{array}\right\}+\left\{\begin{array}{c}
t_{x \mathrm{f}}(s) \\
t_{z \mathrm{f}}(s) \\
w_{\mathrm{f}}(s)
\end{array}\right\}\right) \mathrm{d} s=0 .
$$

Substituting equation (9) into equation (13) results in

$$
\boldsymbol{T}_{\mathrm{p}}\left\{\begin{array}{l}
p(s) \\
r(s) \\
q(s)
\end{array}\right\}=\boldsymbol{T}_{\mathrm{f}},
$$

where

$$
\boldsymbol{T}_{\mathrm{p}}=\int_{\mathrm{s}} \boldsymbol{W}^{\mathrm{T}}(s) \boldsymbol{g}_{t \mathrm{u}}(s) \mathrm{d} s
$$

and

$$
\boldsymbol{T}_{\mathrm{f}}=-\int_{\mathrm{s}} \boldsymbol{W}^{\mathrm{T}}(s)\left\{t_{x \mathrm{f}}(s), t_{z \mathrm{f}}(s), w_{\mathrm{f}}(s)\right\}^{\mathrm{T}} \mathrm{d} s .
$$

Combining the solutions of equations (14) and (8), the surface displacements become

$$
\left\{\begin{array}{l}
U_{x} \\
U_{z}
\end{array}\right\}=\left\{\begin{array}{l}
U_{x \mathrm{f}} \\
U_{z \mathrm{f}}
\end{array}\right\}+\boldsymbol{g}_{u \mathrm{u}}(s) \boldsymbol{T}_{\mathrm{p}}^{-1} \boldsymbol{T}_{\mathrm{f}}
$$

It should be noted that, the conditions for zero 
stress of a drained or undrained boundary at the surface of the half-space are satisfied automatically, since the free-field response and the dynamic Green's functions of layered half-space are obtained under the corresponding boundary conditions.

\section{Verification of accuracy}

To verify the accuracy of the method of this study, we compare our results with those by Liang and Liu (2009), for the surface displacement amplitude of a homogeneous half-space (with a circular cavity) of dry poroelastic medium or saturated poroelastic ones with drained or undrained boundaries. The parameters for the poroelastic medium are described
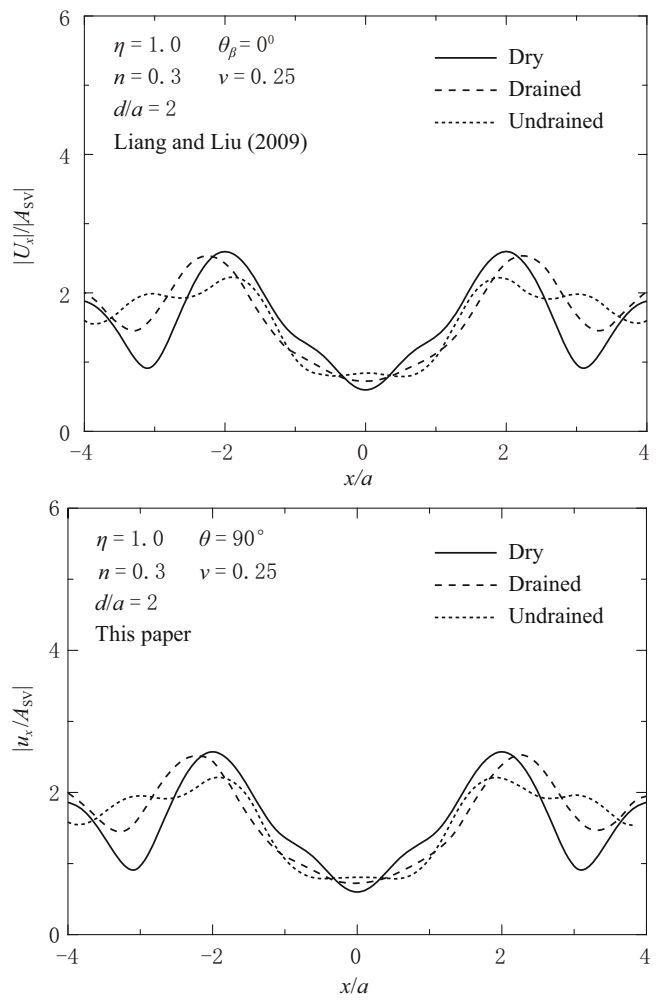

by porosity $n=0.3$, shear modulus $\mu=100 \mathrm{MPa}$, mass density of solid grain $\rho_{\mathrm{s}}=2.65 \times 10^{3} \mathrm{~kg} / \mathrm{m}^{3}$, mass density of fluid $\rho_{\mathrm{f}}=1.0 \times 10^{3} \mathrm{~kg} / \mathrm{m}^{3}$, and Biot's parameters $M=164 \mathrm{MPa}, m=7.219 \times 10^{3} \mathrm{~kg} / \mathrm{m}^{3}$ and $\alpha=0.83$; other parameters are Poisson's ratio $\nu=0.25$, and damping ratio $\zeta=0.001$. These parameters are identical to those normalized ones in Liang and Liu (2009). The cavity is featured with buried depth $d / a=2$, where $a$ is the radius of the cavity. A dimensionless frequency $\eta=(\omega a / \pi) \sqrt{\rho_{\mathrm{s}} / \mu}$ is defined, where $\omega$ is the circular frequency of incident wave. It can be seen from Figure 2 that our results are in good agreement with those by Liang and Liu (2009) for dry poroelastic medium and saturated poroelastic ones with drained and undrained boundaries.
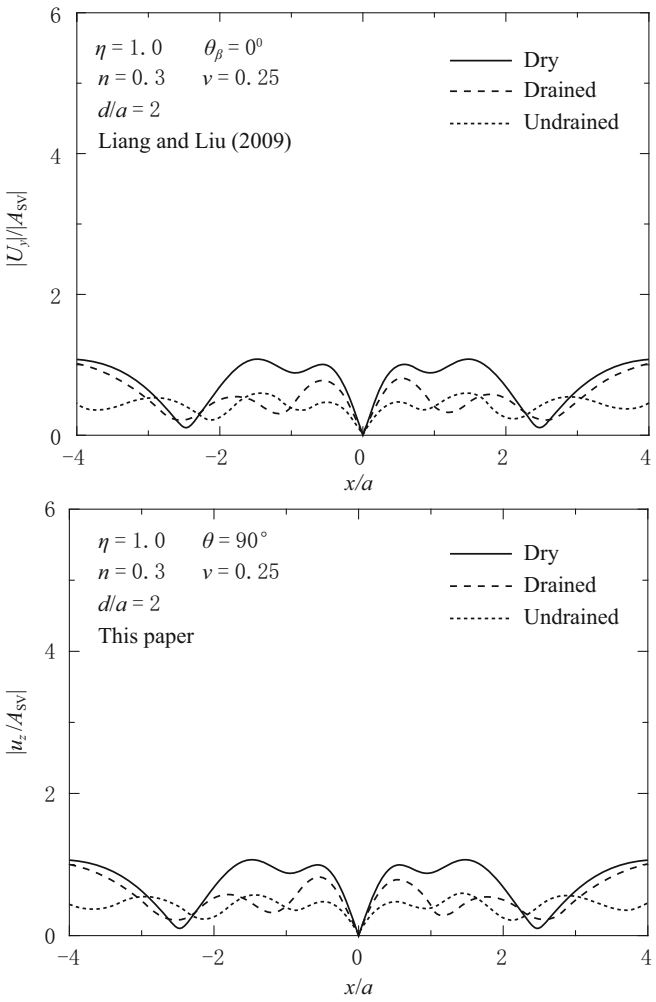

Figure 2 Comparison between surface displacement around a cavity of this study (lower panels) and those by Liang and Liu (2009) (upper panels).

\section{Numerical results and analyses}

The amplification of seismic ground motion due to two cavities is numerically studied for a typical poroelastic layered site in Tianjin. The parameters for the dry poroelastic soil layers, saturated poroelastic soil layers, and for the elastic bedrock are shown in Table 1. The boundary between the dry and the saturated soil layers and between the adjacent saturated soil layers are drained; while the boundary between the saturated soil layer at the bottom and the bedrock and the boundaries on the surface of the cavities are undrained. Both two cavities are featured with radius $a=5 \mathrm{~m}$ and depth $d=10 \mathrm{~m}$.

For the purpose to explore the influence of pore liquid on the amplification of seismic ground motion by group cavities and for convenient comparison, the geometry parameters for cavities and the elastic parameters for poroelastic soil layers are taken the same as those in Liang et al. (2012b). 
Table 1 Soil parameters of dry and saturated poroelastic soil layers and elastic bedrock

\begin{tabular}{crcccccccc}
\hline Layers & $h / \mathrm{m}$ & $v_{\text {dry }} /\left(\mathrm{m} \cdot \mathrm{s}^{-1}\right)$ & $\rho_{\text {dry }} /\left(10^{3} \mathrm{~kg} \cdot \mathrm{m}^{-3}\right)$ & $\zeta$ & $\rho_{\mathrm{f}} /\left(10^{3} \mathrm{~kg} \cdot \mathrm{m}^{-3}\right)$ & $M / \mathrm{MPa}$ & $\mathrm{m} /\left(10^{3} \mathrm{~kg} \cdot \mathrm{m}^{-3}\right)$ & $\alpha$ & $b /\left(10^{3} \mathrm{Ns} \cdot \mathrm{m}^{-4}\right)$ \\
\hline 1 & 5 & 150 & 1.750 & 0.05 & - & - & - & - & - \\
2 & 5 & 150 & 1.750 & 0.05 & 1.0 & 651 & 7.222 & 0.83 & 1.0 \\
3 & 10 & 175 & 1.775 & 0.05 & 1.0 & 867 & 7.222 & 0.83 & 1.0 \\
4 & 10 & 200 & 1.800 & 0.05 & 1.0 & 1103 & 7.222 & 0.83 & 1.0 \\
5 & 10 & 250 & 1.850 & 0.05 & 1.0 & 1610 & 7.222 & 0.83 & 1.0 \\
6 & 10 & 300 & 1.900 & 0.05 & 1.0 & 2134 & 7.222 & 0.83 & 1.0 \\
7 & 10 & 350 & 1.950 & 0.05 & 1.0 & 2645 & 7.222 & 0.83 & 1.0 \\
8 & 10 & 400 & 2.000 & 0.05 & 1.0 & 3123 & 7.222 & 0.83 & 1.0 \\
9 & 10 & 450 & 2.050 & 0.05 & 1.0 & 3556 & 7.222 & 0.83 & 1.0 \\
Bedrock & $\infty$ & 500 & 2.100 & 0.02 & - & - & - & - & - \\
\hline
\end{tabular}

Note: $h$ denotes thickness of soil layer, $v_{\text {dry }}$ denotes shear velocity of soil layer while it is dry, $\rho_{\text {dry }}$ denotes mass density of soil layer while it is dry; $\zeta$ is damping ratio, $\rho_{\mathrm{f}}$ is mass density of fluid; $M, m$ and $\alpha$ are Biot's parameters, $0 \leqslant M<\infty, 0 \leqslant \alpha \leqslant 1 ; b$ is also a Biot's parameter and $b=0$ if there is no internal friction.

\subsection{Frequency-domain analysis}

We first study spectral amplification of surface acceleration in frequency domain. Figures 3-6 show the spectral amplification of surface acceleration at positions $x / a=0,1,2,3,4,5,6,7,8,9,10$ and 15 , due to two cavities in poroelastic layered half-space for $B / a=2.5,5,10$ and 20 , respectively, with $x$-axis expressing the dimensionless frequency and corresponding true frequency, and with $y$-axis the ratio of the surface acceleration to the bedrock acceleration. A dimensionless frequency is defined as $\eta=\omega a / \pi \bar{c}_{\mathrm{s}}$, where $\omega$ is the circular frequency of the excitation, $\bar{c}_{\mathrm{S}}$ is the averaged shear velocity of the poroelastic soil layers where the cavities are located, and $\bar{c}_{\mathrm{s}}=162.5 \mathrm{~m} / \mathrm{s}$ for the soil properties in Table 1. For comparison, Figure 7 shows the spectral amplification of surface acceleration with only single cavity.

It can be observed from Figure $3(B / a=2.5)$ that, there are two large peaks for $x$-spectral amplification at $x / a=0,1,2,3,4,5$ and 6 , with the lower-frequency $(\eta=0.071$ or $f \approx 1.0 \mathrm{~Hz}$ ) peak located around the resonant frequency of the free-field response and the freefield response taking more percentage in the peak, and with the higher-frequency $(\eta=0.140$ or $f \approx 2.5 \mathrm{~Hz}$ ) peak including more contribution from the scattering and diffraction of waves around two cavities. The lowerfrequency peak and higher-frequency peak are 2.41 and 2.44 , respectively, which are amplified by $11.1 \%$ and $12.4 \%$ compared with 2.17 (for $\eta=0.066$ ), the peak of the free-field response. For other observation points at $x / a=7,8,9,10$ and 15 , there is only one large peak around the first resonant frequency of the free field,
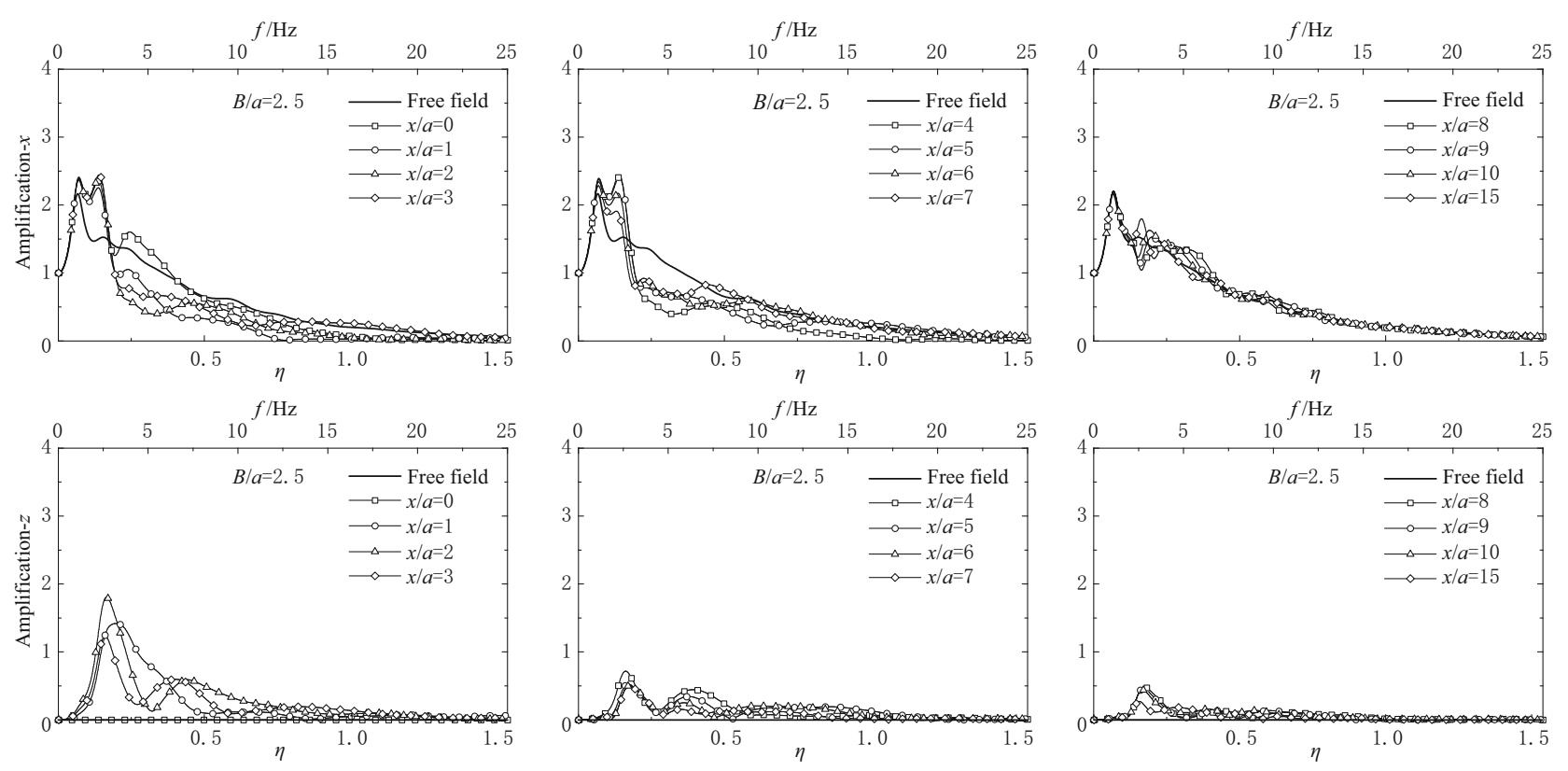

Figure 3 Spectral amplification of surface displacement in the case of two cavities for $B / a=2.5$. 

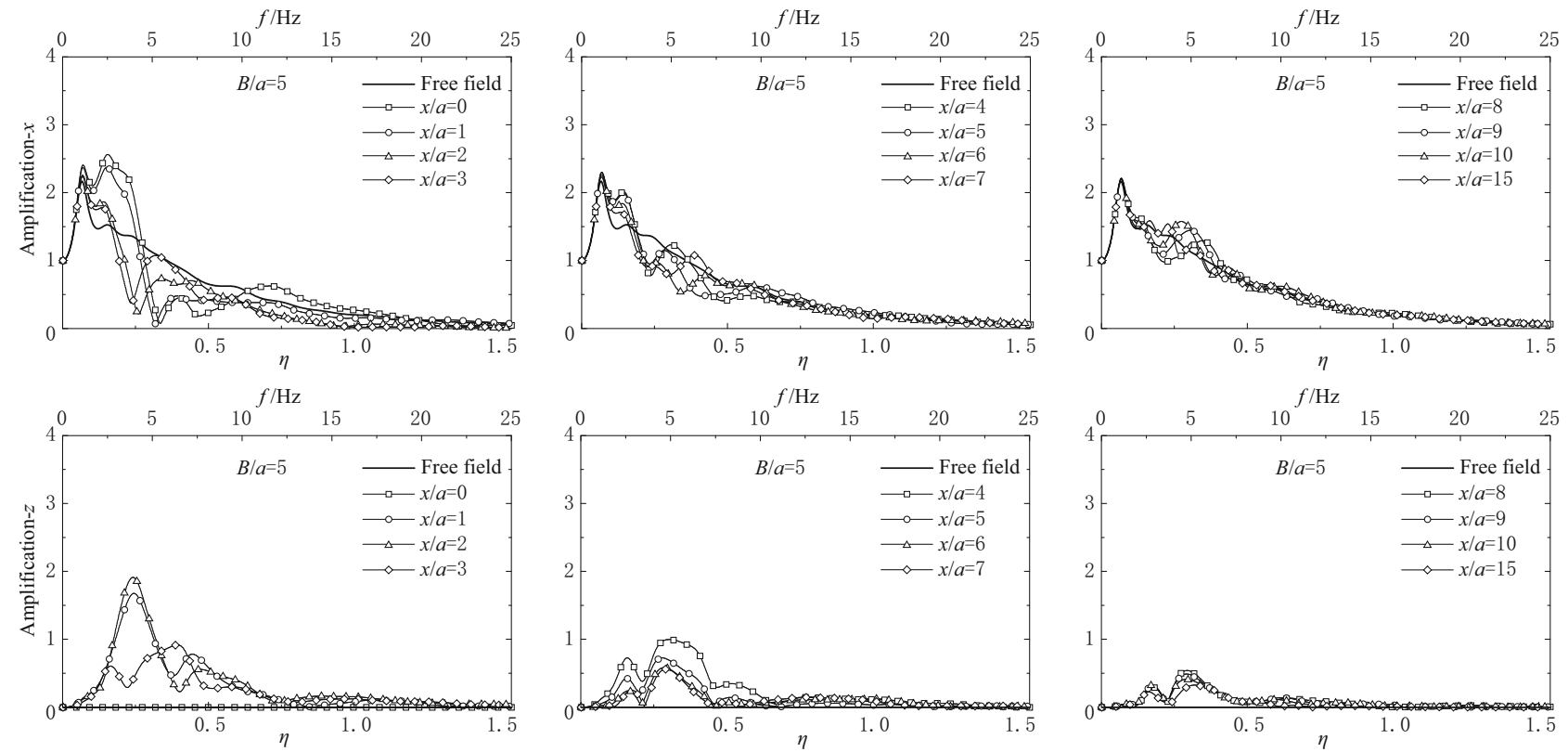

Figure 4 Spectral amplification of surface displacement in the case of two cavities for $B / a=5$.
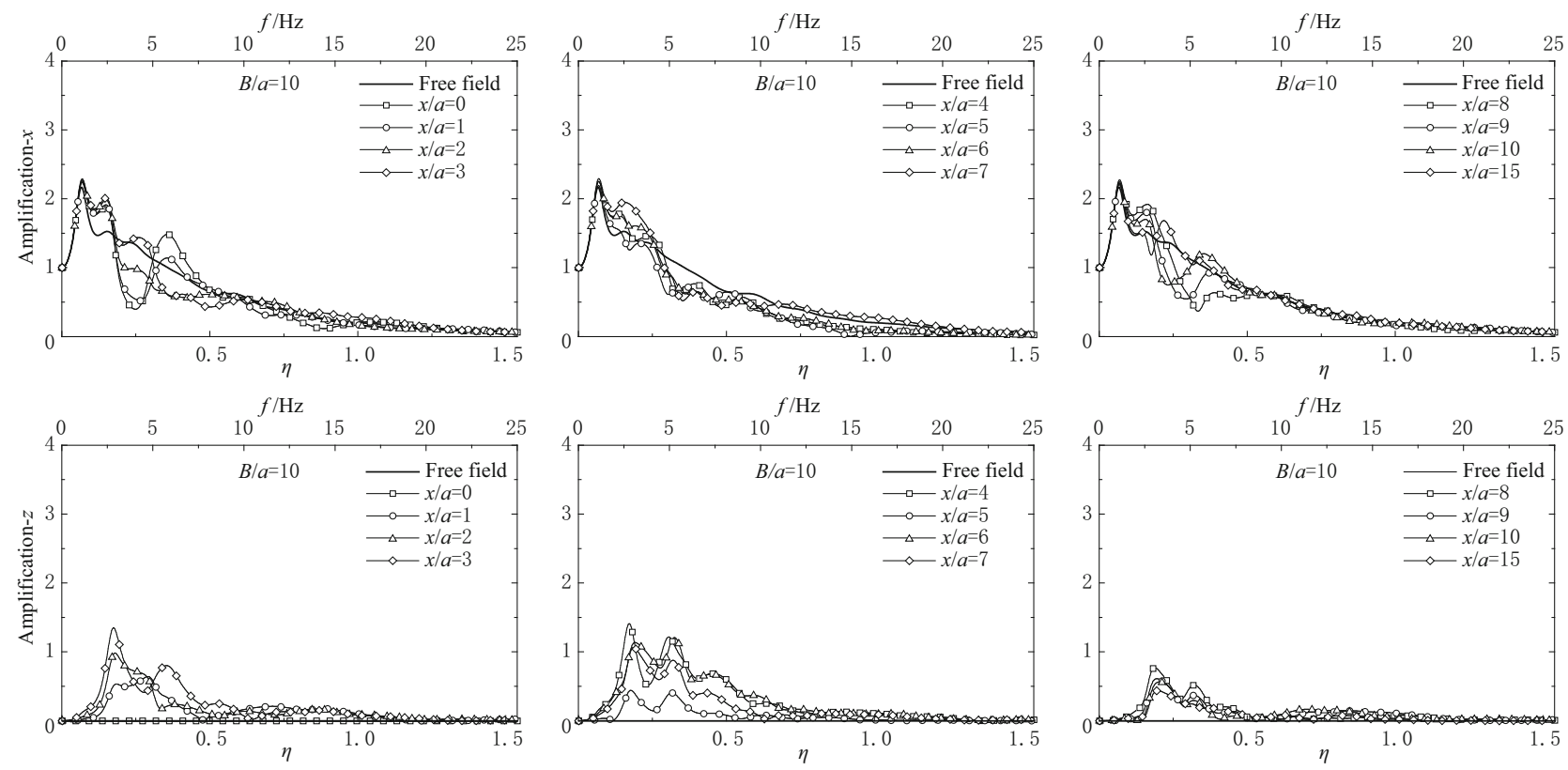

Figure 5 Spectral amplification of surface displacement in the case of two cavities for $B / a=10$.

since these observation points are relatively further from two cavities. It can be shown that, the overlap of the scattering and diffraction of waves around two cavities and the free-field response makes larger amplification of seismic ground motion and wider frequency band of the amplification.

While for $z$-spectral amplification curve, there is only one large peak, which is induced by the scattering and diffraction of waves around two cavities, with the maximum peak 1.80 (for $\eta=0.166$ ) at $x / a=2$, which is enlarged by $48.8 \%$ compared with 1.21 , the peak in the case of single cavity (Figure 7 ). It can be known that there may be large interaction between two cavities.

It can also be found that, the maximum peak (2.44 at $x / a=3)$ in the poroelastic case for $x$-spectral amplification is smaller than that (2.89 at $x / a=3)$ in the 

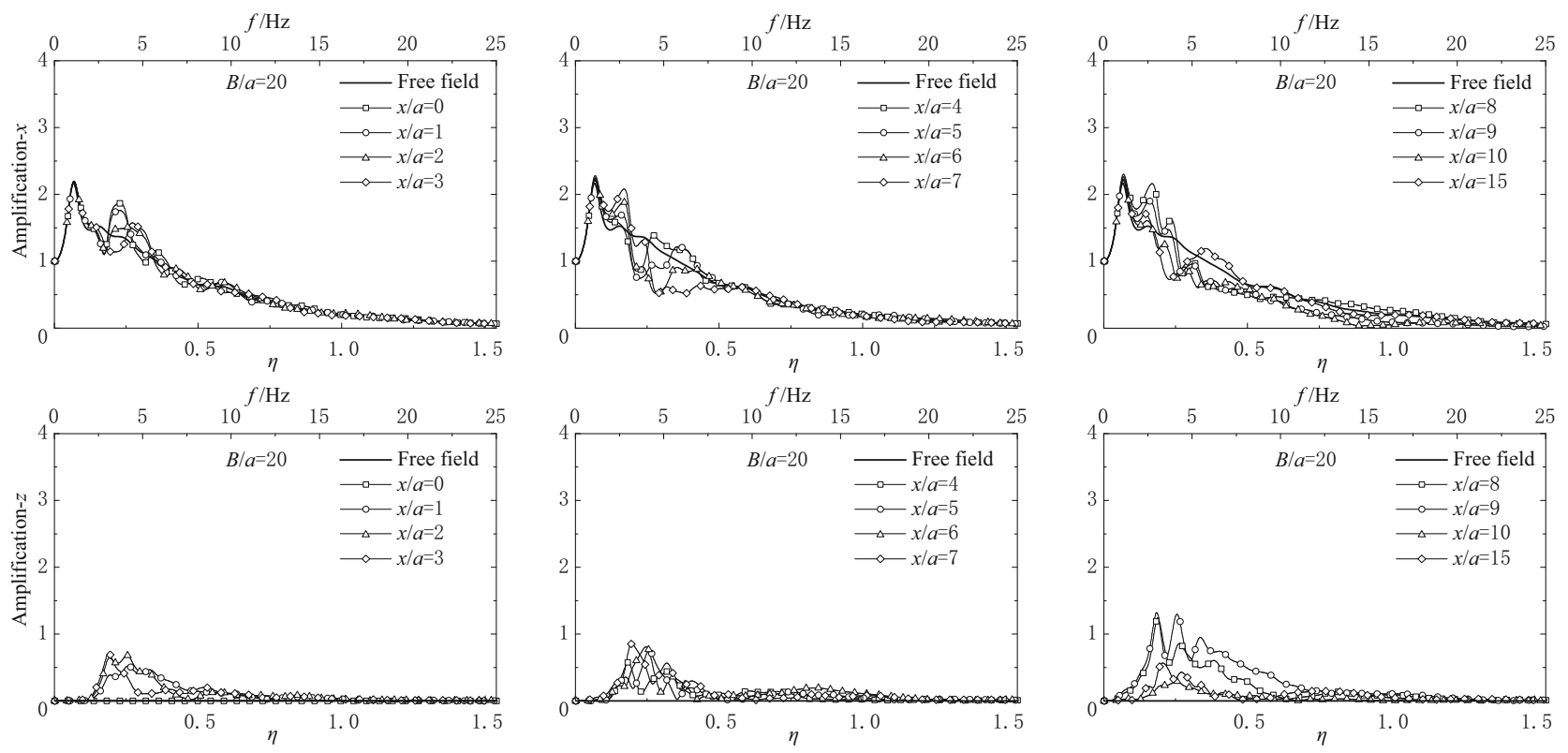

Figure 6 Spectral amplification of surface displacement in the case of two cavities for $B / a=20$.
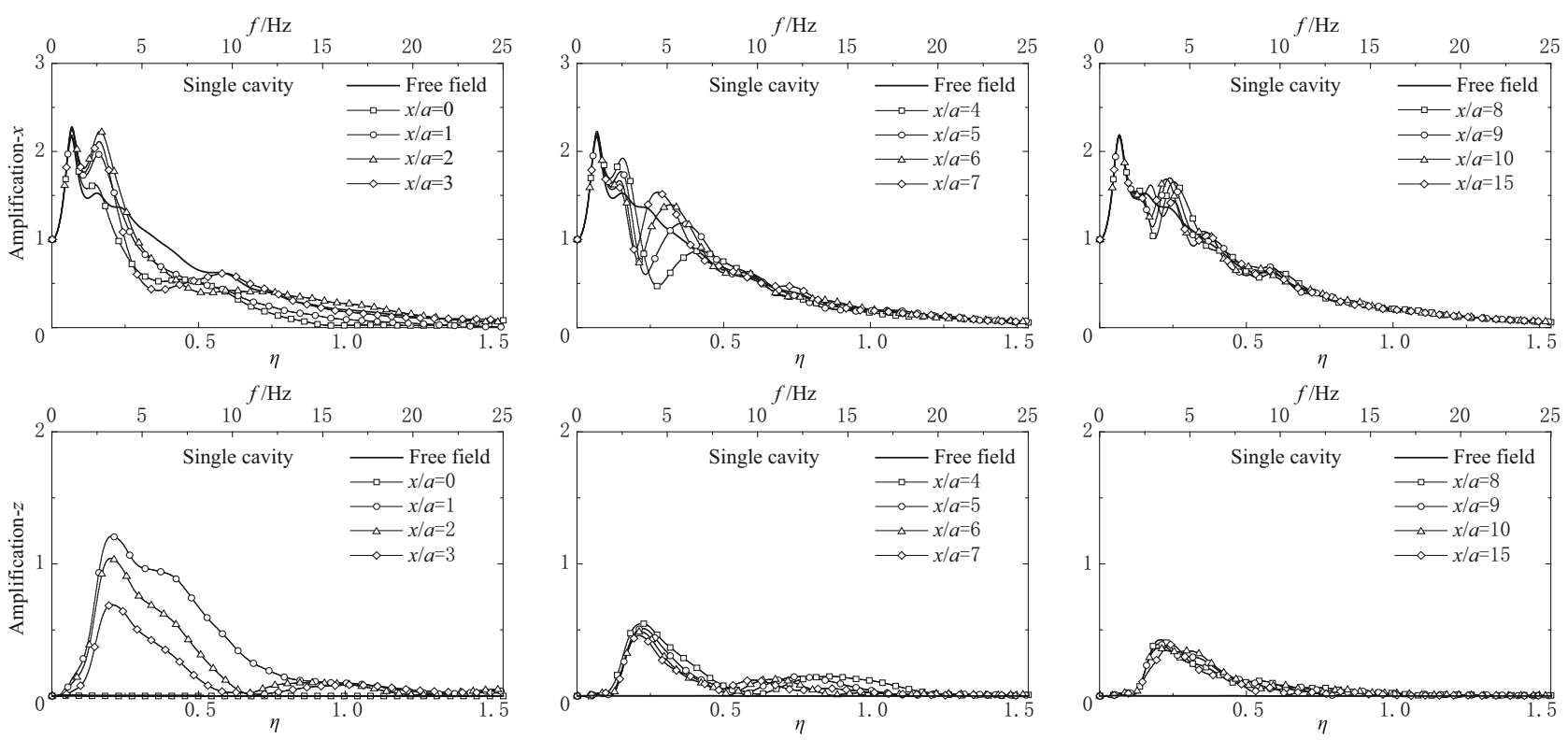

Figure 7 Spectral amplification of surface displacement in the case of single cavity.

elastic case (Liang et al., 2012b), however, the resonant frequency $(\eta=0.140)$ corresponding to the maximum peak is higher than that $(\eta=0.128)$ in the elastic case. It should be pointed that, the resonant frequency $(\eta=0.066)$ of the free-field response in the poroelastic case is almost identical to that $(\eta=0.069)$ in the elastic case (Liang et al., 2012b). For $z$-spectral amplification, the peak is 1.80 (at $x / a=2$ ), much smaller than that (3.11 at $x / a=2)$ in the elastic case (Liang et al.,
$2012 b)$, however, the resonant frequency $(\eta=0.166)$ corresponding to the peak is higher than that $(\eta=0.146)$ in the elastic case. It can be concluded that, the pore liquid may reduce the amplification and increase the frequency of the amplification, both for $x$ - and $z$-spectral amplifications, which may be due to the internal friction between the pore fluid and the solid frame and due to the undrained boundaries.

In the case of $B / a=5$ (Figure 4 ), it can be seen 
that, there are two large peaks for $x$-spectral amplification at $x / a=0$ and 1 , and they are 2.41 (for $\eta=0.071$ ) and 2.56 (for $\eta=0.155$ ), respectively, which are amplified by $11.1 \%$ and $18.0 \%$ compared with the peak of the free-field response, and the higher-frequency peak becomes larger and the frequency band of the large peaks becomes wider than that in the case of $B / a=2.5$. The maximum peak for $z$-spectral amplification is 1.912 for $\eta=0.241$, and the amplitude becomes larger and the frequency for the maximum peak becomes higher, compared with the case of $B / a=2.5$. It can be concluded that large interaction may be induced by certain cavity interval.

In the cases of $B / a=10$ (Figure 5) and $B / a=20$ (Figure 6), there is only one large peak in most cases, with the peaks located around the resonant frequency of the free-field response, and the maximum peak is smaller than that in the cases of $B / a=2.5$ and $B / a=5$. It can be known that the interaction between two cavities becomes smaller as the cavity interval becomes larger.

\subsection{Time-domain analysis}

For time-domain analysis, Taft wave and El Centro wave with peak acceleration of $0.1 \mathrm{~g}$ are used as the ex- citation at the bedrock with the incident angle $\theta=90^{\circ}$. Figure 8 is the acceleration time histories of Taft wave and El Centro wave. Figure 9 illustrates the envelopes of the surface PGAs (peak ground accelerations) under the excitation of Taft wave and El Centro wave, for $B / a=2.5,3,4,5,6,7,8,9,10$ and 20, and Figures 10-11 illustrate the acceleration time histories with the maximum values of the first two extreme PGAs and their response spectra. For the purpose of comparison, Figure 12 shows the envelopes of the surface PGAs for the case of single cavity, and Figures 13-14 show the acceleration time histories with the maximum PGAs and their response spectra.

It can be seen from Figures 9 and 12 that, in the case of two cavities for Taft wave, the maximum horizontal PGA is $0.204 \mathrm{~g}$ at $x / a=0$ for $B / a=4$, and the amplification is up to $38.8 \%$ compared with the PGA (0.147 g) of the free-field response. While in the case of two cavities for El Centro wave, the maximum horizontal PGA is $0.185 \mathrm{~g}$ at $x / a=0$ for $B / a=3$, and the amplification can be $23.3 \%$, compared with the PGA $(0.150 \mathrm{~g})$ of the free-field response. For vertical PGA, the maximum value is $0.090 \mathrm{~g}$ and $0.113 \mathrm{~g}$
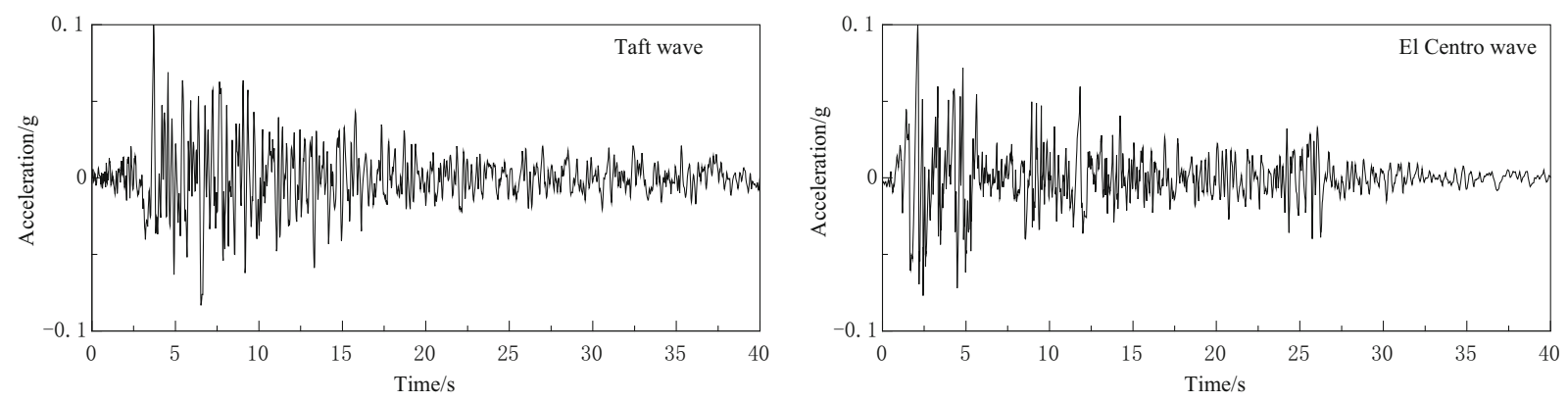

Figure 8 Incident Taft wave and El Centro wave.
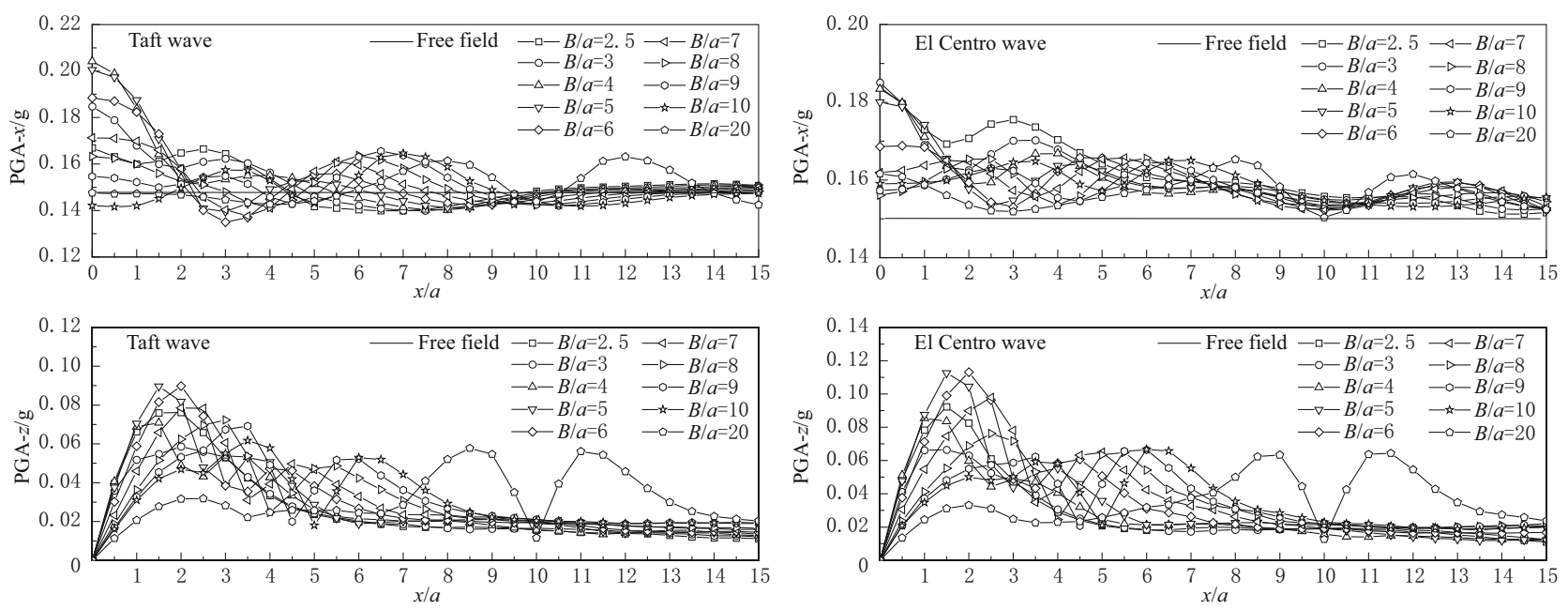

Figure 9 Envelope of PGAs in the case of two cavities. 

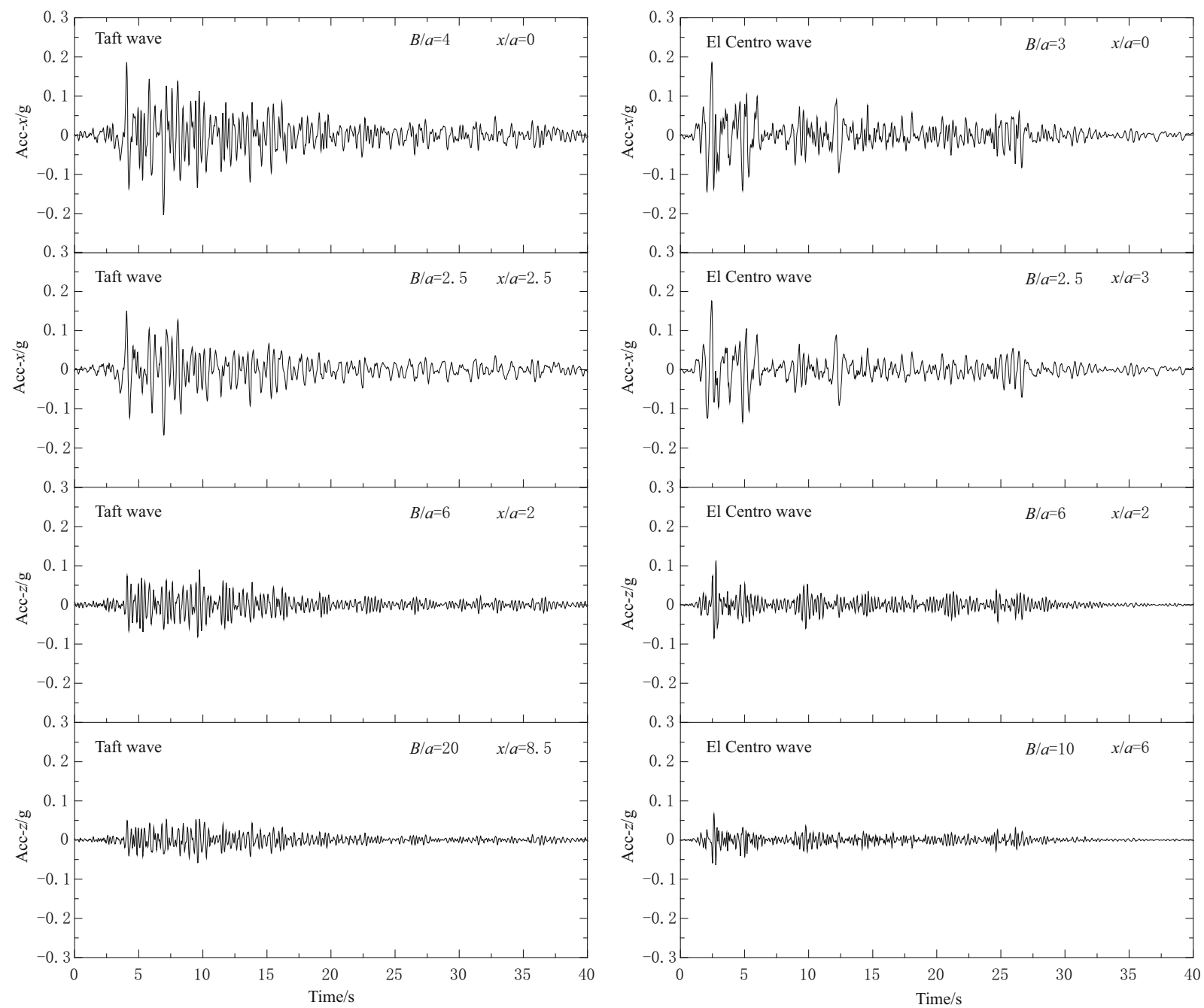

Figure 10 Acceleration time histories in the case of two cavities.

in the case of two cavities for Taft wave and El Centro wave, respectively, both at $x / a=2$ for $B / a=6$. Also it can be seen that the amplification in the case of two cavities is much larger than that in the case of single cavity.

So it can be concluded that group cavities can significantly amplify the seismic ground motion, but the amplification is slightly smaller than the elastic case (Liang et al., 2012b).

It can be observed from Figures 10-11 and 1314 that, in the case of two cavities for Taft wave, the horizontal PRS (peak response spectrum) is $0.836 \mathrm{~g}$ at $x / a=0$ for $B / a=4$ and $0.802 \mathrm{~g}$ at $x / a=2.5$ for $B / a=2.5$, and the amplifications are up to $64.6 \%$ and $57.9 \%$, compared with the PRS $(0.508 \mathrm{~g})$ of the free field. Both the predominant periods of the two response spectra are $0.44 \mathrm{~s}$, identical to that of the response spectrum of the free field. While in the case of two cavities for El Centro wave, the horizontal PRS is $0.567 \mathrm{~g}$ at $x / a=0$ for $B / a=3$ and $0.560 \mathrm{~g}$ at $x / a=3$ for $B / a=2.5$, and the amplifications are $36.6 \%$ and $34.9 \%$, compared with the free-field PRS $(0.415 \mathrm{~g})$. The predominant periods of the two response spectra are $0.52 \mathrm{~s}$ and $0.46 \mathrm{~s}$, different from the predominant period $(0.56 \mathrm{~s})$ of the free-field response spectrum. For vertical response spectra in the case of two cavities for Taft wave, the peak values are $0.438 \mathrm{~g}$ at $x / a=2$ for $B / a=6$ and $0.334 \mathrm{~g}$ at $x / a=8.5$ for $B / a=20$, and both the predominant periods of the response spectra are $0.33 \mathrm{~s}$; while in the case of two cavities for El Centro wave, the peak values are $0.435 \mathrm{~g}$ at $x / a=2$ for $B / a=6$ and $0.241 \mathrm{~g}$ at $x / a=6$ for $B / a=10$, and both the predominant periods of the response spectra are $0.26 \mathrm{~s}$. 

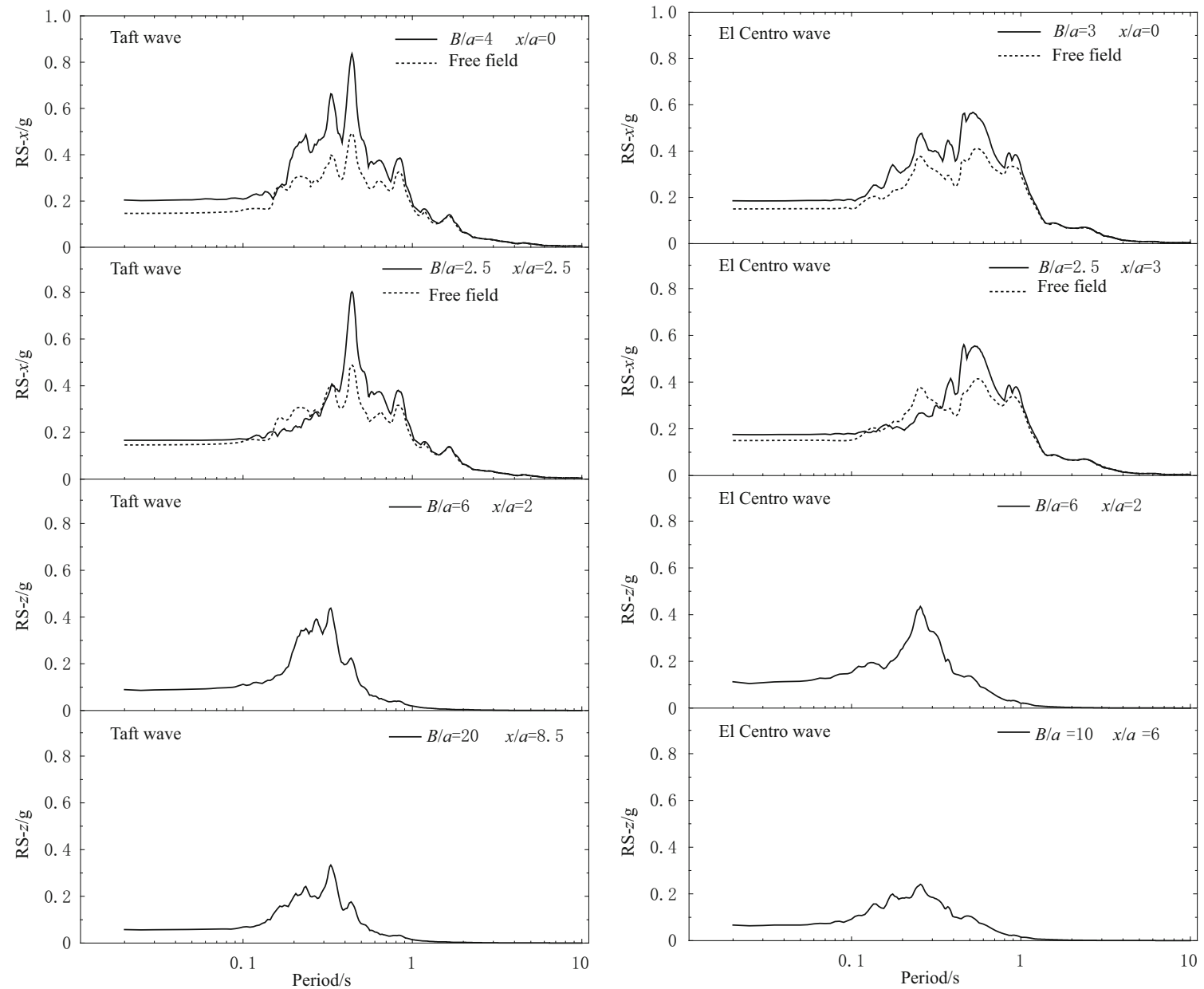

Figure 11 Acceleration response spectra (RS) in the case of two cavities.
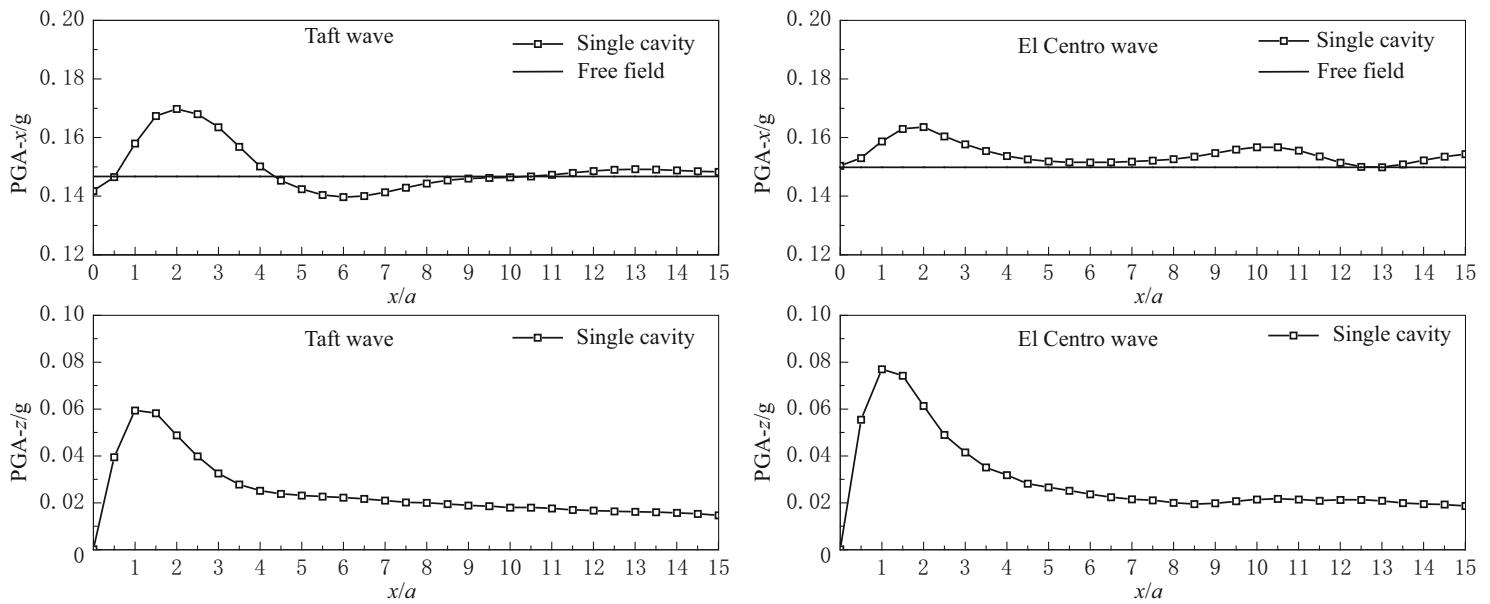

Figure 12 Envelope of PGAs in the case of single cavity.

Therefore, it can be concluded that group cavities can affect the spectra of the seismic ground motion, and the predominant periods of response spectra in poroe- lastic case become shorter to some extent compared with those in the elastic case (Liang et al., 2012b). 

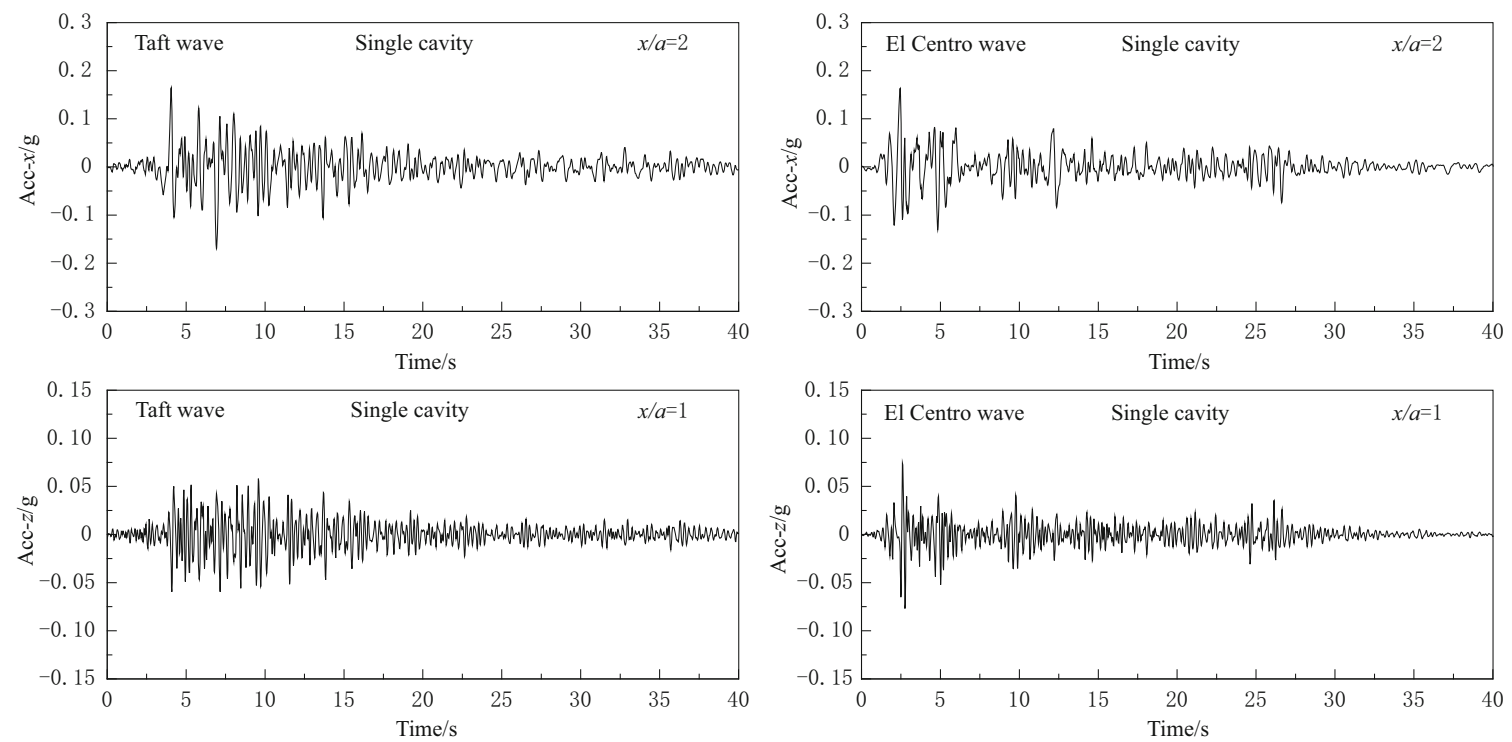

Figure 13 Acceleration time histories in the case of single cavity.
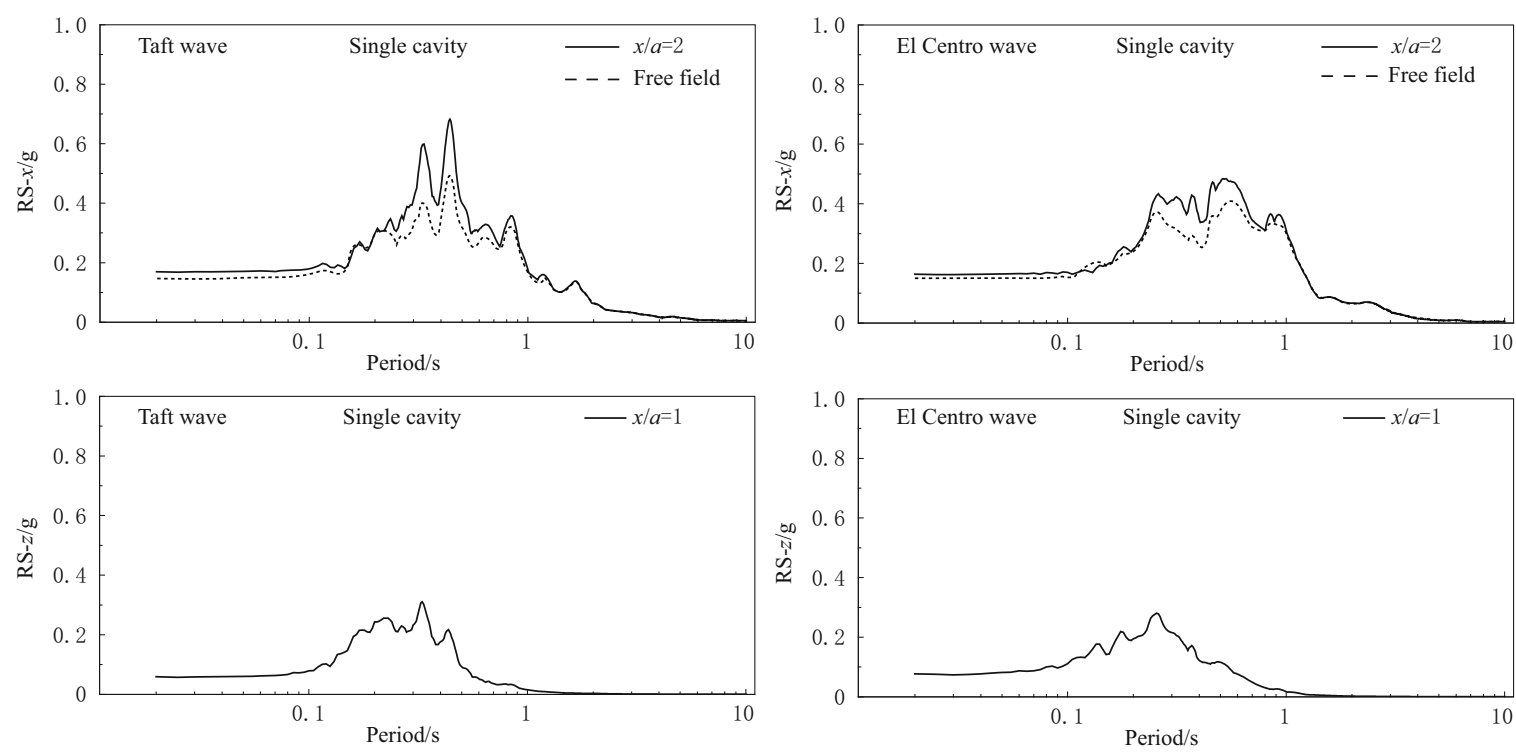

Figure 14 Acceleration response spectra (RS) in the case of single cavity.

\section{Conclusions}

This paper studies amplification of in-plane seismic ground motion by group cavities in layered half-space with dry and saturated poroelastic soil layers using indirect boundary element method (IBEM), both in frequency domain and time domain. It is shown that amplification of seismic ground motion in poroelastic case is slightly smaller than that in the elastic case, and predominant period of response spectra in poroelastic case becomes shorter to some extent compared with that in the elastic case. It is suggested that the effect of underground group cavities in coastal area on design seismic ground motion should be considered in seismic microzonation.

Acknowledgements This study was supported by National Natural Science Foundation of China under grant No. 50978183 and Key Project for Applied Basic Research of Tianjin Municipality under Grant No. 12JCZDJC29000. 


\section{References}

Biot M A (1941). General theory of three-dimensional consolidation. Journal of Applied Physics 12(2): 155-164.

Biot M A (1962). Mechanics of deformation and acoustic propagation in porous media. Journal of Applied Physics 33(4): $1482-1498$.

Liang J W and Liu Z X (2009). Diffraction of plane SV waves by a cavity in poroelastic half-space. Earthquake Engineering and Engineering Vibration 8(1): 29-46.

Liang J W and You H (2004). Dynamic stiffness matrix of a poroelastic multi-layered site and its Green's functions. Earthquake Engineering and Engineering Vibration 3(2):
$273-282$.

Liang J W and You H (2005). Green's functions for uniformly distributed loads acting on an inclined line in a poroelastic layered site. Earthquake Engineering and Engineering Vibration 4(2): 233-241.

Liang J W, Zhang J and Ba Z N (2012a). Time-domain amplification of seismic ground motion by group cavities in layered half-space. China Civil Engineering Journal 45(S1): 152-157 (in Chinese with English abstract).

Liang J W, Zhang J and Ba Z N (2012b). Amplification of in-plane seismic ground motion by group cavities in layered half-space (I). Earthquake Science 25(6): 275-285. 